\title{
Determining the Effective Cutoff Wavelength of Single-Mode Fibers: An Interlaboratory Comparison
}

\author{
DOUGLAS L. FRANZEN, MEMBER, IEEE
}

\begin{abstract}
The National Bureau of Standards (NBS), in cooperation with the Electronic Industries Association, conducted an interlaboratory measurement comparison among six fiber manufacturers to determine the effective cutoff wavelength of single-mode fibers. Measurement techniques based on transmitted power were used to determine cutoff wavelength on four fibers designed for single-mode operation at $1300 \mathrm{~nm}$. NBS also contributed results using a spectral near-field technique. One standard deyiation measurement spreads for the various techniques range from 6 to $12 \mathrm{~nm}$. With the appropriate data analysis, single bend attenuation and power step methods give the same results. Both techniques are easily implemented as extensions to the usual spectral attenuation measurement.
\end{abstract}

\section{INTRODUCTION}

$\mathrm{C}$ UTOFF WAVELENGTH of the second-order $\mathrm{LP}_{11}$ mode is an important parameter for single-mode fibers. Practical systems are operated close to the cutoff wavelength to enhance fundamental mode confinement, but sufficiently far from cutoff so that second-order mode power is not transmitted. Significant amounts of second-order mode power are undesirable since multiple pulses arising from differential mode delays reduce system bandwidth, and interference between first- and second-order modes results in modal noise at fiber joints.

Theoretical $\mathrm{LP}_{11}$ cutoff wavelength as determined from the refractive index profile is difficult to experimentally measure and is not of interest here. For practical needs, the concept of an "effective" cutoff wavelength is useful and gives an easyto-measure quantity. Effective cutoff wavelength is the wavelength above which second-order mode power is below a given level. It, therefore, depends on fiber length and curvature, and is usually determined by some form of spectral transmission measurement.

The National Bureau of Standards, in cooperation with the Electronic Industries Association (EIA), conducted an interlaboratory measurement comparison to determine effective cutoff wavelength. ${ }^{1}$ Participants included NBS and six fiber manufacturers. Tested were three transmission methods; two of which could be easily implemented along with a spectral attenuation measurement. NBS, in addition, supplied results using a near-field method.

Manuscript received July $13,1984$.

The author is with the National Bureau of Standards, Electromagnetic Technology Division, Boulder, CO 80303.

${ }^{1}$ Comparisons were conducted in cooperation with EIA Subcommittee P6.6.5.
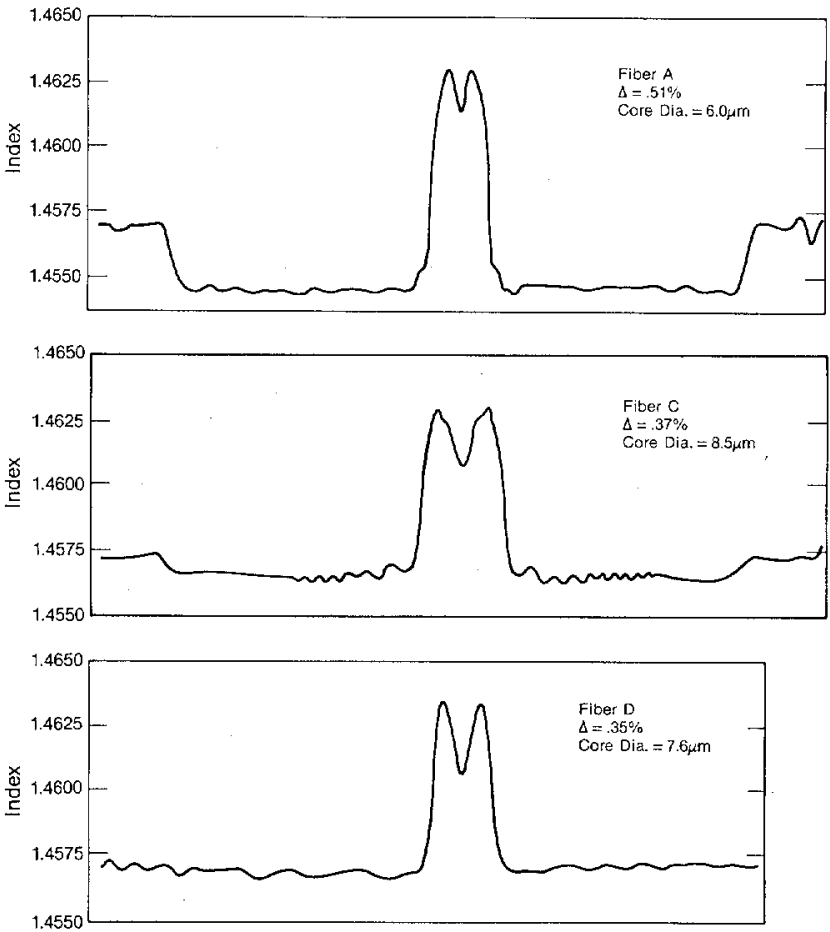

Fig. 1. Index of refraction profiles determined by refracted near-field scanning for the comparison fibers. Delta is the contrast given approximately as $\left(n_{\text {core }}-n_{\text {cladding }}\right) / n_{\text {core }}$ for an equivalent step-index profile. Fiber $B$ was not extensively used and was therefore not measured.

Four fibers designated $A, B, C$, and $D$ were used in the comparisons. The fibers represent typical commercial products designed for single-mode telecommunications at $1300 \mathrm{~nm}$. Included are a diverse mix of index profile designs (Fig. 1). Fiber $A$ has a depressed inner cladding with a relatively small core and high contrast, fiber $C$ has a more mildly depressed inner cladding, and fiber $D$ has a matched (single) cladding.

Effective cutoff wavelength determined from a transmission method depends on the relative amount of second-order mode power detected; hence, values are a function of fiber length and curvature. A typical variation with length for a specific fiber design is shown in Fig. 2. Fig. 2 gives the change in effective cutoff wavelength with respect to a 5-m sample length; cutoff decreases as length increases. Plots such as Fig. 2, determined for a particular design or class of fiber, allow cutoff to be estimated for any length once a 5 -m sample has been measured. Effective cutoff is found to decrease as a logarithmic 


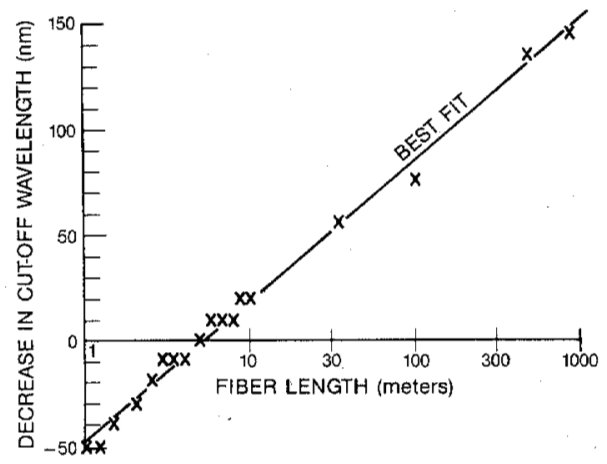

Fig. 2. Typical length dependence of effective cutoff wavelength. Change with respect to a 5 -m fiber length (AT\&T Bell Laboratories data).

function of length [1]. In this comparison, all participants determined effective cutoff wavelength for a $3.0 \pm 0.2-\mathrm{m}$ fiber length. Also, for experimental convenience, the measurements were made with a single $60-\mathrm{cm}$-diam loop in the test fiber. This eliminates the need to maintain a straight fiber on the test bench and improves measurement reproducibility by placing a minimum bend radius in the test sample, i.e., other unintentional bends will be of larger curvature and presumably will not affect the measurements.

\section{Measurement Methods}

\section{A. Single Bend Attenuation}

This method determines cutoff wavelength by measuring the spectral attenuation of a single small diameter loop in the fiber. At cutoff, the second-order mode power is loosely bound to the core and is easily attenuated by a tight bend. Bend attenuation is determined by measuring first the spectral power transmitted through the unperturbed "straight" sample ${ }^{2} P_{s}(\lambda)$ then wrapping a single turn around a mandrel and measuring the transmitted spectral power $P_{b}(\lambda)$. Attenuation of the bend in decibels is given by

$$
10 \log \frac{P_{s}(\lambda)}{P_{b}(\lambda)} \text {. }
$$

Cutoff is defined as the wavelength where the long-wavelength edge of the attenuation has increased by $0.1 \mathrm{~dB}$ over the longwavelength baseline (Fig. 3). Cutoff determined by this method is relatively insensitive to mandrel diameter (Figs. 4 and 5). Too small a mandrel results in fundamental mode attenuation at longer wavelengths, whereas too large a mandrel gives poor reproducibility. Figs. 4 and 5 show the bend attenuation for fibers $A$ and $D$ for mandrels of $16,20,30$, and $50 \mathrm{~mm} \mathrm{diam}^{3}$. The long-wavelength edge does not exhibit much change; for fiber $D$ a $16 \mathrm{~mm}$ diam is probably too small and gives a slight shift in the cutoff wavelength. The two fibers show different degrees of fundamental mode confinement determined in part by the core/clad contrast. For comparisons, participants, submitted cutoff results using both $20-$ and $30-\mathrm{mm}$-diam mandrels.

\footnotetext{
${ }^{2}$ In our case a single $60-\mathrm{cm}$-diam loop. Bend mandrels were placed approximately at fiber mid-points.

3 A small wavelength correction (monochromator calibration) is necessary before absolute values can be extracted from the NBS curves.
}

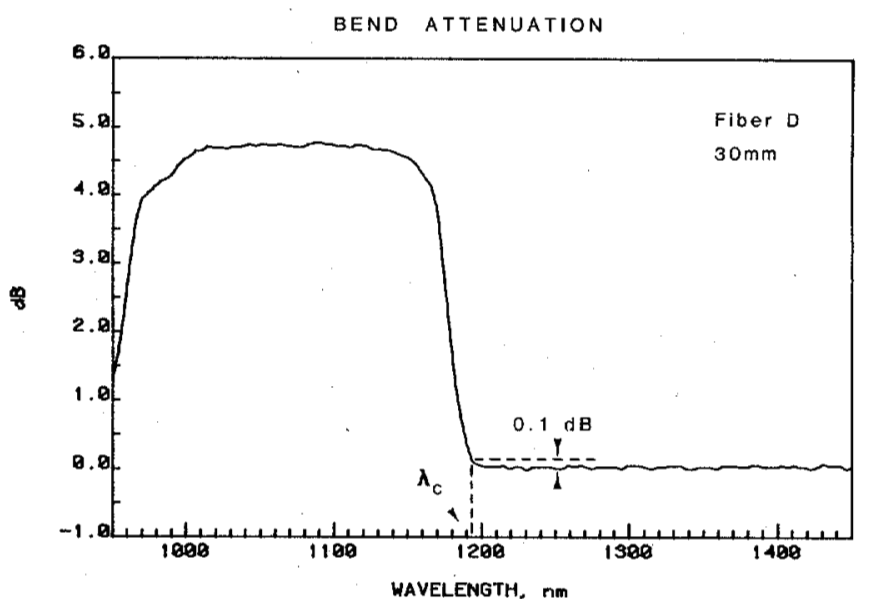

Fig. 3. Effective cutoff wavelength determined by the single bend attenuation method. Cutoff is defined as the wavelength where the attenuation edge has increased by $0.1 \mathrm{~dB}$ over the long-wavelength baseline (fiber $D, 30$-mm-diam mandrel).

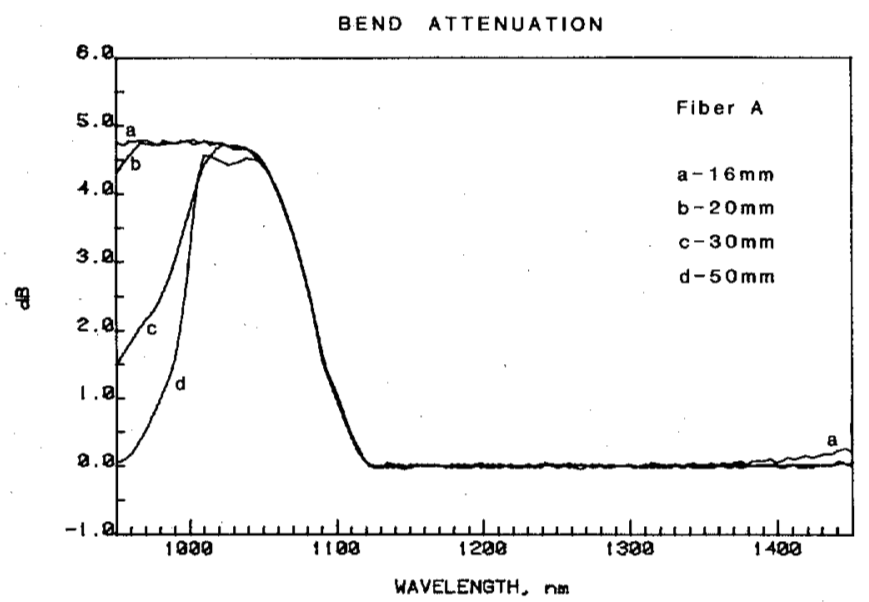

Fig. 4. Single bend attenuation with mandrel diameter as a parameter (fiber $A$ ). Long-wavelength attenuation edge is unchanged with respect to mandrel diameter.

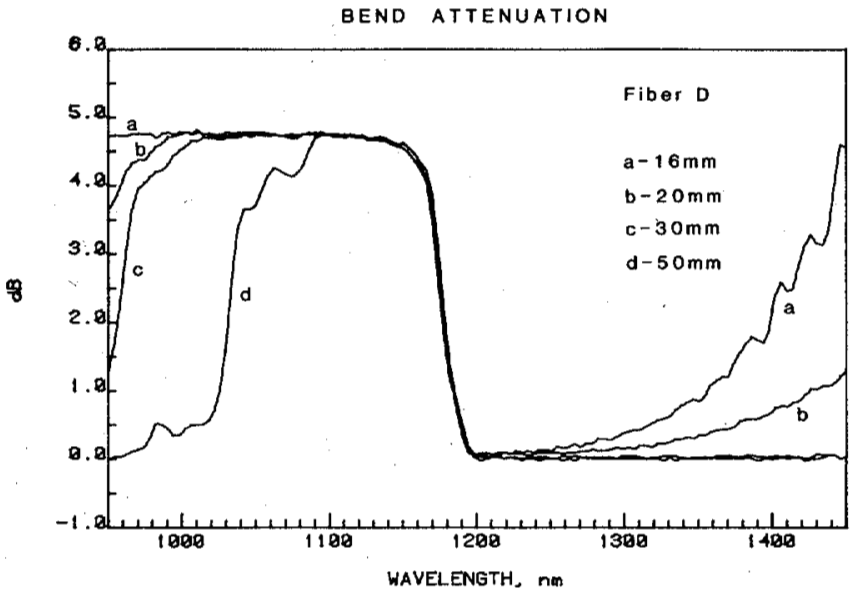

Fig. 5. Single bend attenuation with mandrel diameter as a parameter (fiber $D$ ). Fundamental mode is not as well confined as in fiber $A$. A $16-\mathrm{mm}$-diam mandrel is too small and results in a small shift of the long-wavelength attenuation edge.

\section{B. Power Step}

When a fiber is illuminated with a spectrally flat source, the transmitted power will show an abrupt increase as cutoff is 


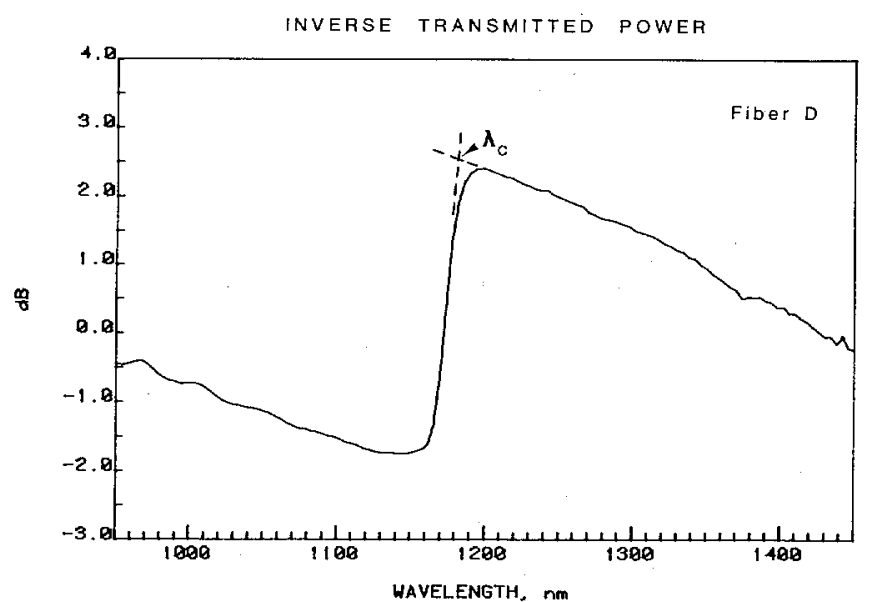

Fig. 6. Effective cutoff wavelength determined by the power step method (inverse of the transmitted power plotted). Spectral power exiting the single-mode fiber is referenced to a multimode fiber. Cutoff is defined using the tangent intercept point.

approached from the long-wavelength side. This is due to the addition of second-order mode power. Theoretically, since the second-order mode has twice the degeneracy of the fundamental, the received power should increase by a factor of three $(4.8 \mathrm{~dB})$. For these comparisons, participants actually determined the inverse of the transmitted spectral power given by

$$
10 \log \frac{P_{m}(\lambda)}{P_{s}(\lambda)}
$$

where $P_{m}(\lambda)$ is the spectral power transmitted through a short length of multimode fiber and $P_{s}(\lambda)$ is the spectral power transmitted through the single-mode fiber under test. Normalizing the power to a multimode fiber eliminates possible large spectral variations in system response, e.g., source, grating, order-sorting filters, detector, etc. For this method, cutoff was defined at the tangent intercept point where, from the long-wavelength side, the inverse power shows an abrupt decrease (Fig. 6). Procedural definitions for this method and the other methods were not chosen arbitrarily but represent current practice for various manufacturers.

\section{Multiple Bend}

Effective cutoff wavelength depends on fiber curvature. Decreasing the radius of curvature causes cutoff to shift to shorter wavelengths. This method determines cutoff wavelength as a function of fiber curvature [2]. Curvature is placed in the fiber using a single turn around a mandrel. Cutoff wavelength for each curvature is determined from the transmitted spectral power ratio

$$
\frac{P_{b}(\lambda)}{P_{m}(\lambda)}
$$

where $P_{b}(\lambda)$ is the spectral power transmitted through the bent single-mode fiber and, for purposes of normalization, $P_{m}(\lambda)$ is the spectral power transmitted through a multimode fiber ${ }^{4}$. Cutoff is the wavelength where the tangent to the

\footnotetext{
${ }^{4}$ As with the other methods, a single 60 -cm-diam loop remains in the
} fiber.

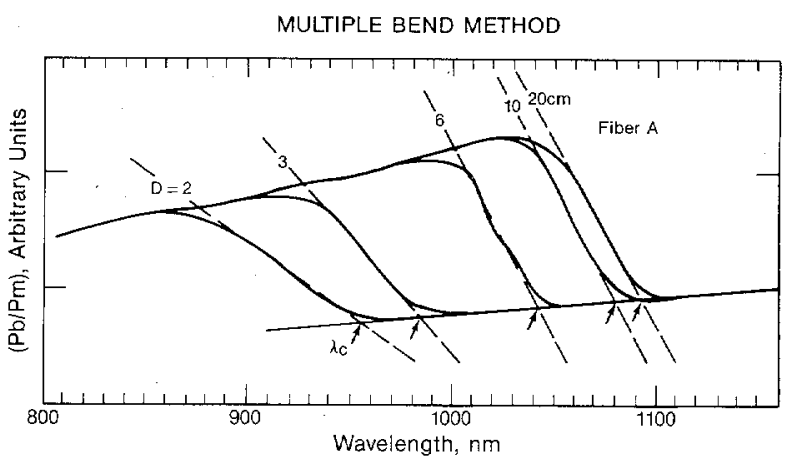

Fig. 7. Effective cutoff wavelength determined by the multiple bend method. Cutoff wavelength is determined as a function of fiber curvature. Shown are a family of transmitted power curves giving cutoff wavelengths $\lambda_{c}$, for curvature diameters of $2,3,6,10$, and 20 cm (Corning Glass Works data, fiber $A$ ).

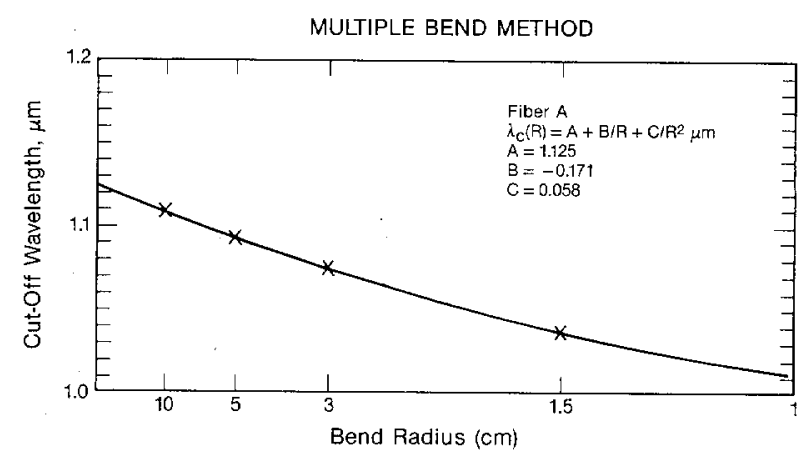

Fig. 8. Effective cutoff wavelengths from the multiple bend method fit to the polynomial expression $\lambda_{c}(R)=A+(B / R)+\left(C / R^{2}\right)(\mathrm{AT} \& \mathrm{~T}$ Bell Laboratories data, fiber $A$ ).

power step intersects the base line (Fig. 7). Fig. 7 shows a family of curves resulting from mandrel diameters of $2,3,6$, 10 , and $20 \mathrm{~cm}$ and the associated cutoff wavelengths for each bend curvature. Participants were asked to plot cutoff wavelength versus bend radius or determine coefficients to the following power series expansion

$$
\lambda_{c}(R)=A+\frac{B}{R}+\frac{C}{R^{2}}
$$

where $\lambda_{c}$ is the cutoff wavelength; $R$ is the bend radius; and $A$, $B$, and $C$ are constants. Fig. 8 is a typical example showing (4) fit to the experimental points from a measurement on fiber $D$. The cutoff wavelength determined by this technique for comparison against the other measurement techniques is given by $\lambda_{c}(30 \mathrm{~cm})$.

\section{Near-Field Intensity Pattern}

Fundamental and second-order modes have different radial intensity variations across the fiber core. The fundamental mode is nearly Gaussian shaped while the second-order mode is zero on-axis. As cutoff is approached from long wavelengths, the addition of second-order mode power causes the near-field profile to progress from having an on-axis peak to an on-axis minimum (Fig. 9). The wavelength where the profile is flat has been used as a measure of cutoff [3]. NBS implemented this technique at long wavelengths and contributed measurements using this definition of cutoff wavelength. Fig. 9 shows 


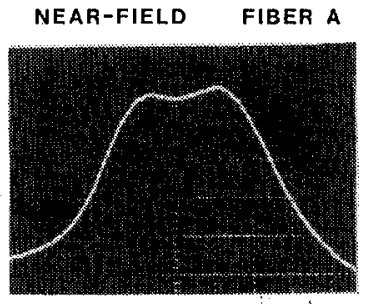

$1088 \mathrm{~nm}$

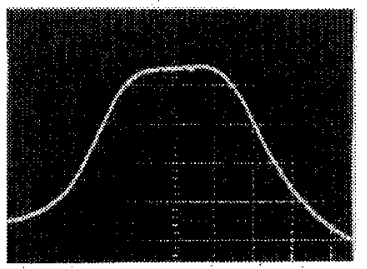

$1098 \mathrm{~nm}$

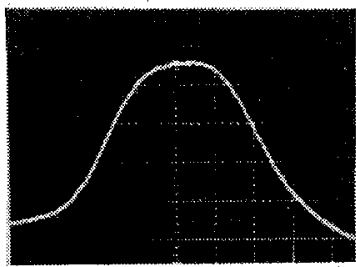

$1108 \mathrm{~nm}$

Fig. 9. Effective cutoff wavelength determined by observing the spectral near-field intensity profile. Cutoff is the wavelength where the near-field intensity has a "flat top" which in this example for fiber $\boldsymbol{A}$ is $1098 \mathrm{~nm}$ (middle curve). The measurement system is continuously tunable in wavelength and gives a near-field intensity profile observable on an oscilloscope at a repetition rate of $2 \mathrm{~Hz}$.

typical data obtained with the system which uses a torque motor to scan a magnified near-field image across a multimode fiber pigtailed to a liquid nitrogen cooled germanium detector. The flat portion of the near-field profile can be established with a wavelength uncertainty of approximately $\pm 10 \mathrm{~nm}$.

\section{RESULTS}

Participants were provided with two sample lengths of each fiber. Samples were cut from different parts of the fiber so each participant received a piece from the beginning and one from the end. This was done to determine possible length dependence of local cutoff wavelength; such an effect could result from a taper in core diameter and has been previously observed [4]. Such an effect did not exist in the comparison fibers. Fig. 10 shows cutoff wavelength determined by single bend attenuation plotted as a function of adjacent sample number; there is no apparent slope to the data.

Results are not given for fiber $B$. This fiber was silicone buffered and some participants reported difficulty in stripping the cladding light using their usual techniques. Cladding intensity should be reduced by at least $20 \mathrm{~dB}$ compared to the core to offset the relatively large difference in the cross-sectional area. Insufficient data were reported for fiber $B$ to yield meaningful results. The information that was reported is consistent with a problem in stripping cladding light.

Participants determined cutoff wavelength for two samples of each fiber. Reported values for each sample are the average of two repeated measurements. Figs. 11, 12, and 13 give the results for fibers $A, C$, and $D$ using the single bend attenuation and transmitted power methods. One participant's results for fibers $A$ and $D$ were several standard deviations below the aver-
ÇUT-OFF WAVELENGTH, BEND METHOD, 20mm MANDREL
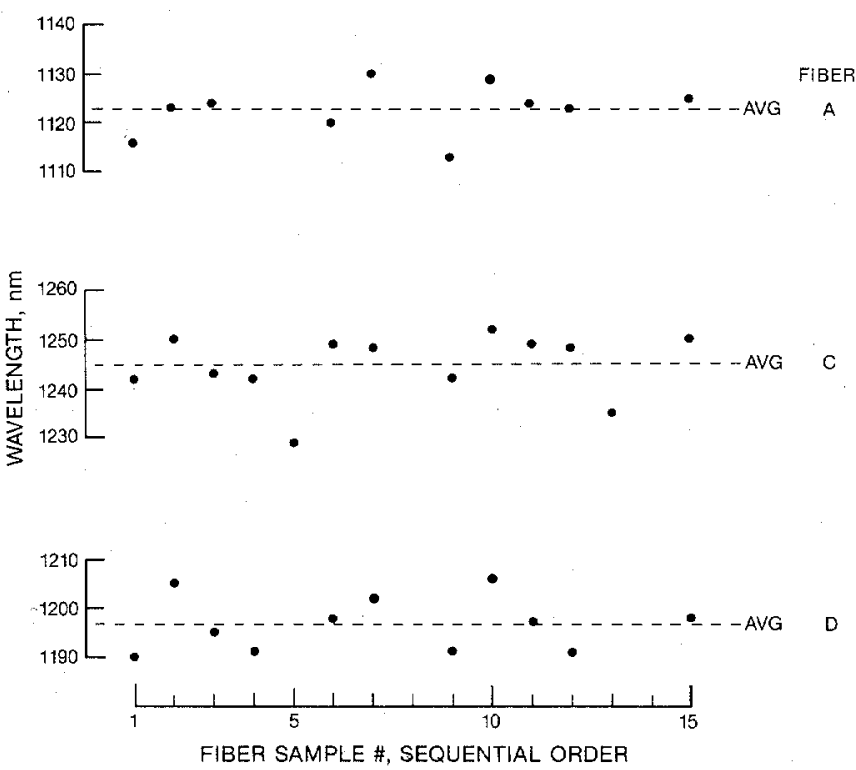

Fig. 10. Effective cutoff wavelength results for fibers $A, C$, and $D$ using the single bend attenuation method plotted as a function of the sequential test sample number. Local cutoff wavelength is not a function of fiber length at the given level of measurement scatter.

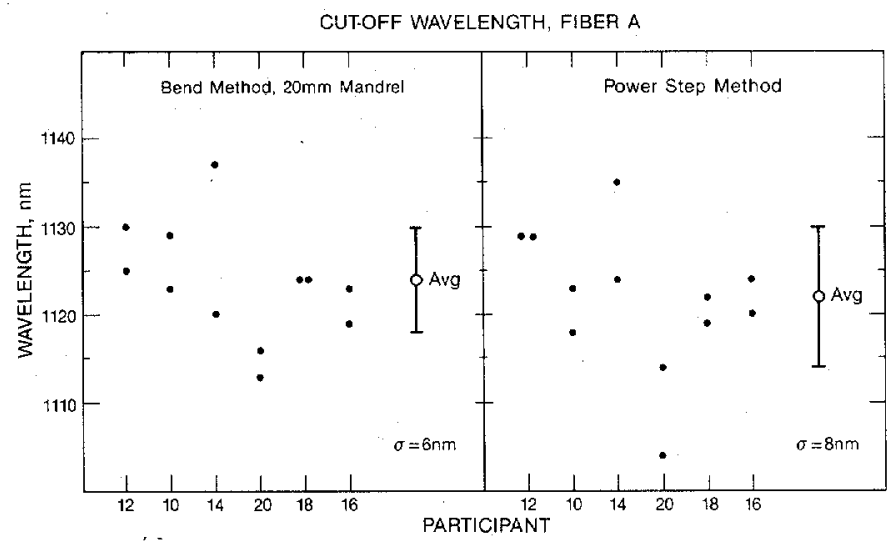

Fig. 11. Comparison results giving effective cutoff wavelength of fiber $A$ using the single bend attenuation and power step methods. One standard deviations are 6 and $8 \mathrm{~nm}$, respectively.

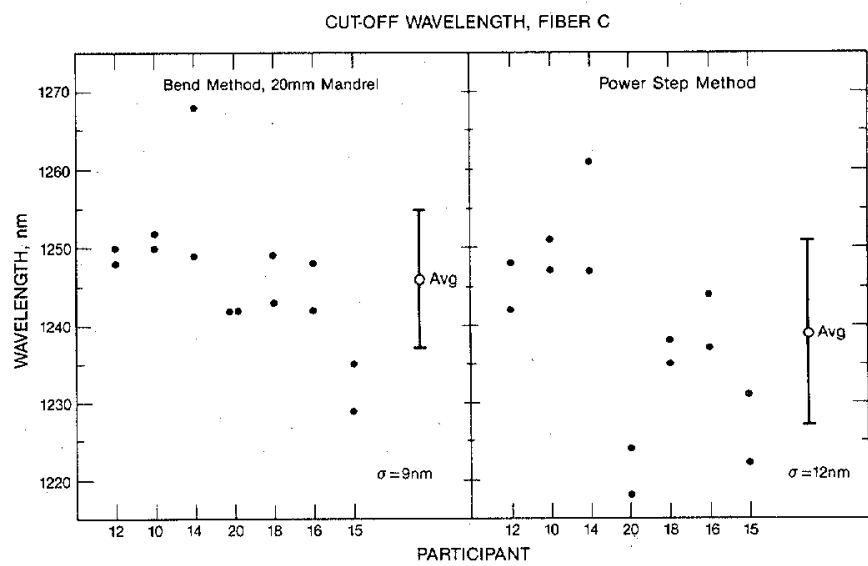

Fig. 12. Comparison results giving effective cutoff wavelength of fiber $C$ using the single bend attenuation and power step methods. One standard deviations are 9 and $12 \mathrm{~nm}$, respectively. 
CUT-OFF WAVELENGTH, FIBER D

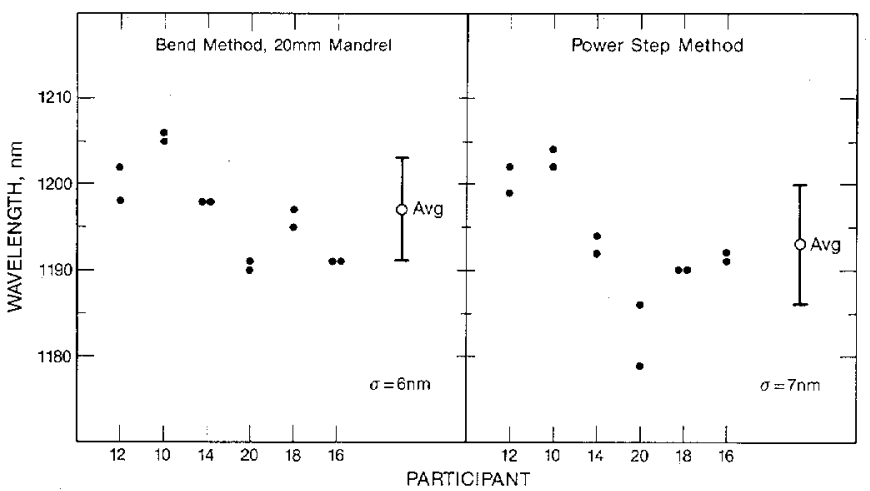

Fig. 13. Comparison results giving effective cutoff wavelength of fiber $D$ using the single bend attenuation and power step methods. One standard deviations are 6 and $7 \mathrm{~nm}$, respectively.

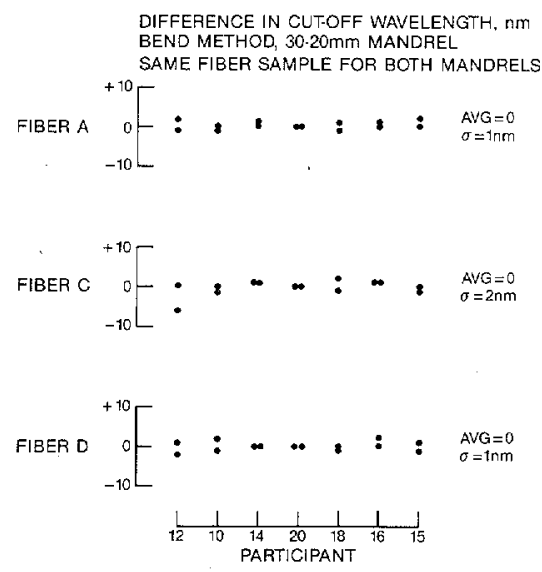

Fig. 14. Single bend attenuation, cutoff wavelength determined using 30-mm-diam mandrel minus cutoff wavelength determined using 20 -mm-diam mandrel. There is statistically no difference.

age; they are considered nonrepresentative and are, therefore, not included. For the single bend attenuation method, one standard deviation spread is 6,9 , and $6 \mathrm{~nm}$ for fibers $A, C$, and $D$, respectively. There is no significant difference in cutoff wavelength between measurements with 20 - and $30-\mathrm{mm}$-diam mandrels (Fig. 14). For the power step method, one standard deviation spread is 8,12 , and $7 \mathrm{~nm}$ for fibers $A, C$, and $D$, respectively. An algorithm for locating curve tangents was not specified. Different subjective judgements in locating the tangents may have increased the measurement spread for the power step method.

Systematic offset between single bend attenuation and power step methods is insignificant for fibers $A$ and $D$ and about one standard deviation for fiber $C$. One participant reports that better agreement between the two methods is possible if the data is reduced in the same manner; e.g., both methods use either the tangent intercept or $0: 1-\mathrm{dB}$ level definition. This is not surprising since in the region of cutoff the two methods yield similar shaped curves. This is best illustrated when single bend attenuation and power step curves are superimposed (Fig. 15) with power step being plotted as

$10 \log \frac{P_{s}(\lambda)}{P_{m}(\lambda)}$.

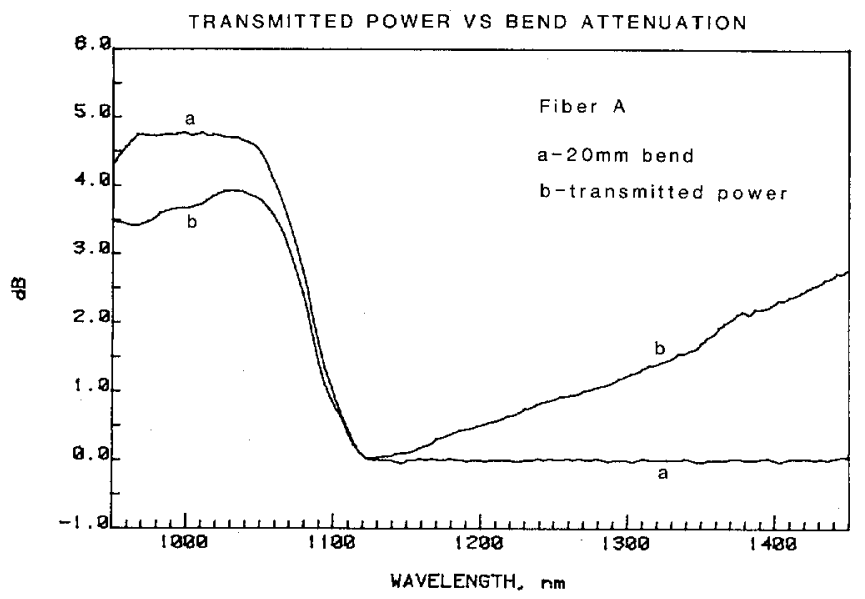

Fig. 15. Superposition of single bend attenuation $a$, and transmitted power (power step) $b$, spectral curves. At the cutoff edge the two curves overlap and have a similar shape over a limited wavelength range (fiber $A$ ).

FIBER C, BEND METHOD

CUT-OFF EDGE DETAIL
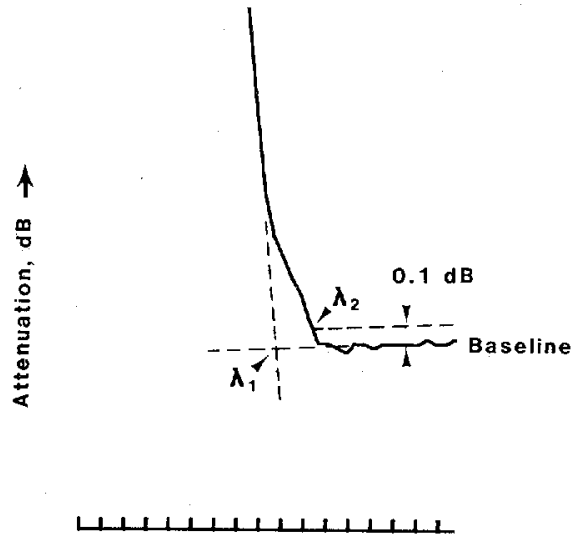

Wavelength Increment, $10 \mathrm{~nm}$

Fig. 16. Detail of the long-wavelength edge of the single bend attenuation for fiber $C$ showing the two methods of data analysis $\lambda_{1}$, tangent intercept and $\lambda_{2}, 0.1-\mathrm{dB}$ level, $\lambda_{2}-\lambda_{1}=15 \mathrm{~nm}$.

In the single bend attenuation method, the output power from the "straight" fiber is compared to the output from the fiber when it is strongly bent. Cutoff wavelength of the bent fiber is shifted to shorter wavelength thus in the vicinity of cutoff, the attenuation curve is not too different from the shape of the transmitted power. Data analysis using tangent intercepts gives a lower value for cutoff wavelength than does the $0.1-\mathrm{dB}$ level. This is most obvious in fiber $C$ which has a more gradual cutoff and a pedestal to the curve. For the NBS measurements, the pedestal magnitude was not very reproducible. Fig. 16 is a measurement where the pedestal is more pronounced. As shown, the tangent intercepts give a $15-\mathrm{nm}$ lower value for cutoff then does the 0.1-dB level. Participants supplied curves from which their cutoff values were taken. The curves were analyzed to determine cutoff using the $0.1-\mathrm{dB}$ level for the power step method on fiber $C$ (Table I). As shown, the offsets are reduced when the $0.1-\mathrm{dB}$ level is used for both methods. 
TABLE I

Cltoff Wavelength $(\mathrm{nm})$ Fiber $\mathrm{C}$ Different Methods of DATA ANALYSIS

\begin{tabular}{|c|c|c|c|c|c|}
\hline Participant & $\begin{array}{c}\# 1 \\
\text { Single Bend, } \\
0.1 \mathrm{~dB} \text { level }\end{array}$ & $\begin{array}{c}\# 2 \\
\text { Power } \\
\text { Step, Tan. } \\
\text { Intercept }\end{array}$ & $\begin{array}{c}\# 3 \\
\text { Power } \\
\text { Step, } \\
\text { O.1 } \\
\mathrm{dB} \text { level }\end{array}$ & $\# 1-\# 2$ & $\# 1-\# 3$ \\
\hline 12 & 1249 & 1245 & 1251 & 4 & -2 \\
\hline 10 & 1251 & 1249 & 1252 & 2 & -1 \\
\hline 14 & 1259 & 1254 & 1260 & 5 & -1 \\
\hline 20 & 1242 & 1221 & 1243 & 21 & -1 \\
\hline 18 & 1246 & 1237 & 1246 & 9 & 0 \\
\hline 16 & 1245 & 1241 & $*$ & 4 & $*$ \\
\hline 15 & 1232 & 1227 & 1231 & 5 & +1 \\
\hline \hline Average & 1246 & 1239 & 1247 & 7 & -1 \\
\hline $\begin{array}{c}\text { Stc. Dev, } \\
0\end{array}$ & 8 & 12 & 10 & & \\
\hline
\end{tabular}

* Could Not Be Accurately Determined From Supplied Data

TABLE II

Multiple Bend Method versus Single Bend Method (nm) (20-mm MANDREL)

\begin{tabular}{|c|c|c|c|c|c|}
\hline FIBER & $\lambda$ mult. & $\begin{array}{c}\text { STD } \\
\text { DEV }\end{array}$ & $\lambda$ single & $\begin{array}{c}\text { STD. } \\
\text { DEV. }\end{array}$ & $\begin{array}{c}\lambda \text { mult. } \\
\lambda \text { single }\end{array}$ \\
\hline A & 1123 & 9 & 1124 & 6 & -1 \\
\hline C & 1247 & 15 & 1246 & 9 & 1 \\
\hline D & 1188 & 28 & 1197 & 6 & -9 \\
\hline
\end{tabular}

The improved agreement is particularly noticeable for participant $\# 20$. A similar analysis for fibers $A$ and $C$ indicates the difference between the average cutoff wavelengths (single bend attenuation minus power step) change from 2 and $4 \mathrm{~nm}$, respectively, to -3 and $-1 \mathrm{~nm}$.

The single bend attenuation and power step methods are important because they are easily implemented along with spectral attenuation measurements. Only one additional measurement is required; consequently, they are likely methods for manufacturing quality control. It is also of interest to determine whether the multiple bend or near-field method give any particular advantage.

The multiple bend method requires several measurements and more detailed data analysis. Table II gives the results for the multiple bend method compared to single bend attenuation. As shown, the measurement spread is larger with agreement between techniques being within one standard deviation. Principal use of the multiple bend method will probably be limited to those situations where it is necessary to determine (4).

Near-field measurement precision is not quite as good as with the other methods. The "flat-top" cutoff feature can be located within approximately $10 \mathrm{~nm}$. Also involved is the subjective opinion of the operator as to when the profile is flat. Table III shows a comparison of near-field cutoff wavelengths to single bend attenuation cutoff wavelength. There is substantial disagreement; this can be explained by the relative amount of second-order mode power utilized by each method. Bend attenuation and power step, as used here, define cutoff when a small amount of second-order mode power is present.
TABLE III

Cutoff Wayelength Comparison: Near-Field Method to Single BEND METHOD (nm)

\begin{tabular}{|c|c|c|c|c|c|}
\hline FIBER & $\begin{array}{c}\text { \#1 } \\
\text { Single Bend } \\
\text { Method } 20 \mathrm{~mm} \\
\text { mandrel, } \\
0.1 \mathrm{~dB} \text { level, } \\
\text { Participant Ave. }\end{array}$ & $\begin{array}{c}\# 2 \\
\text { Single Bend } \\
\text { Method } 20 \mathrm{~mm} \\
\text { mąndrel, } \\
2.6 \text { dB level, } \\
\text { NBS Dafä }\end{array}$ & $\begin{array}{c}\# 3 \\
\text { Near-Field } \\
\text { Methods, } \\
\text { Flat Top, } \\
\text { NBS Data }\end{array}$ & $\# 1-\# 3$ & $\# 2-\# 3$ \\
\hline A & 1124 & 1091 & $1089 \pm 10$ & 35 & +2 \\
\hline C & 1246 & 1217 & $1224=10$ & 22 & -7 \\
\hline
\end{tabular}

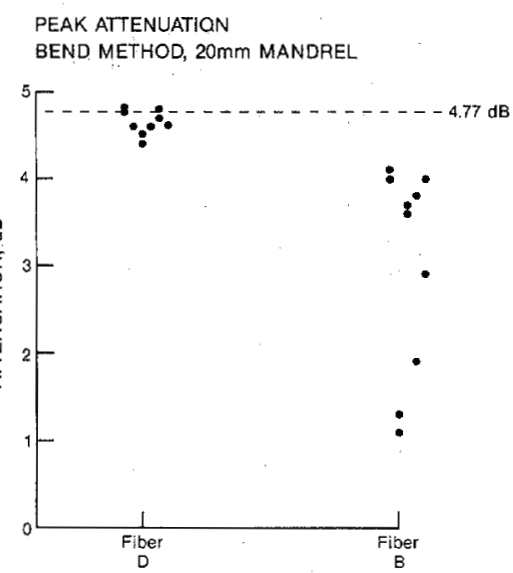

Fig. 17. Peak attenuation values from single bend attenuation curves for fibers $D$ and $B$. Shown ị the theoretical level expected when the second-order mode has twice the power as the fundamental. Increased scatter for fiber $B$ is consistent with residual cladding light.

To flatten out the fundamental mode near-field profile requires a substantial amount of second-order mode power that is approximately 80 percent of the fundamental [5]. When cutoff by the single bend method is determined at the 2.6-dB level (approximately 80-percent second-order mode power), the two methods agree within error limits (Table III). While the near-field method gives cutoff wavelength with a single measurement, the apparatus is rather complex and the fiber end must be carefully imaged.

Several interesting effects are apparent after analyzing the cutoff wavelength data. One is the maximum level of attenuation observed with the bend attenuation method. If the second-order mode carries twice the power as the fundamental, and is completely attenuated by the bend, the attenuation to the short wavelength side of cutoff should increase by 4.77 $\mathrm{dB}$ (factor of 3). Figs. 4 and 5 show the mandrels do completely attenuate the second-order mode after it has been completely established in the "straight" fiber, i.e., smaller diameter mandrels do not further increase the attenuation. Fig. 17 shows the peak attenuation taken from participant's data for fibers $D$ and $B$. Results for fiber $D$ are typical of those for fibers $A$ and $C$. The peak attenuations are, on the average, only a few tenths of a decibel below theoretical expectations. Fiber $B$ exhibits a rather large scatter in peak attenuation. This is consistent with high levels of cladding light present along with first- and second-order mode powers. Since total fiber power is detected, cladding light influences the fractional change of core light with respect to total detected light: It should be pointed out that a determination of cutoff wave- 
TABLE IV

Cutorf Wayelfangth Variation betwfify Different Samples of the SAMi: Fibre, Bfad Method, 20-mm Mandrel.

\begin{tabular}{|c|c|}
\hline FIBER & $\begin{array}{c}\text { AVERAGE ABSOLUTE } \\
\text { DIFFERENCE, nm }\end{array}$ \\
\hline$A$ & $8 \mathrm{~nm}$ \\
\hline$C$ & $6 \mathrm{~nm}$ \\
\hline$D$ & $2 \mathrm{~nm}$ \\
\hline
\end{tabular}

length does not depend on peak attenuation and the method can still be successfully applied to fiber $B$.

For a typical participant, different samples from the same fiber exhibit greater measurement differences than do repeated measurements on the same sample. Cutoff wavelength measurements using single bend attenuation on the same samples with 20- and 30-mm-diam mandrels give an inferred precision of a few nanometers (Fig. 14). Absolute differences between cutoff wavelengths from different samples of the same fiber using single bend attenuation are given in Table IV. The differences are substantially greater than a few nanometers. We presently have no explanation for this effect. It is possible that there are real differences in cutoff wavelength between different samples. On the other hand, differences could arise from changes in the fiber position on the test bench. With different samples, repositioning must naturally occur. For repeated measurements on the same sample, the fiber can be left in relatively the same position.

In conclusion, single bend attenuation and power step methods as tested will give an average precision of approximately $8 \mathrm{~nm}$. Depending on the particular fiber, the systematic offsets are small or comparable to the interlaboratory measurement spread. Offsets can be further reduced by analyzing the curve shapes in a similar manner.

\section{ACKNOWLEDGMENT}

The author would like to thank P. A. Simpson of NBS for his help in designing the NBS Measurement System. NBS acknowledges the cooperation and support of the EIA Subcommittee on single-mode fiber, P6.6.5, chaired by F. Kapron. Also appreciated is the timely cooperation of the following six manufacturers and individuals for participating in the comparisons: A. H. Cherin and W. T. Anderson (AT\&T Bell Laboratories, Norcross, GA), P. R. Reitz and J. M. Dick (Corning Glass Works, Waveguide Products Engineering Laboratory, Corning, NY), M. Dakss and M. P. Singh (GTE, Waltham, MA), F. P. Kapron, C. Ragdale, R. Sheldon, and D. Sharp (ITT, ElectroOptical Products Division, Roanoke, VA), G. J. Cannell (Standard Telecommunication Laboratories, Harlow, UK), and W. C. Meixner and J. Chapman (Valtec, West Boylston, MA).

\section{REFERENCES}

[1] Y. Kitayama and S. Tanaka, "Effective cut-off wavelength of single-mode fiber," in Tech. Dig., OFC 84 (New Orleans, LA), 1984, paper TUN 14. W. T. Anderson and T. A. Lenahan, "Length dependence of the effective cutoff wavelength in single-mode fibers," J. Lightwave Technology, vol. LT-2, pp. 238-242, June 1984.

[2] P. D. Lazay, "Effect of curvature on the cutoff wavelength of single-mode fibers," in Tech. Dig. Symp. Opt. Fiber Measurements, (Nat. Bur. Stand.), 1980, (U.S.) Spec. Publ. 597.

[3] Y. Murakami, A. Kawana, and H. Tsuhiya, "Cut-off wavelength measurements for single-mode optical fibers," Appl. Opt., vol. 18, no. 7, pp. 1101-1104, Apr. 1, 1979.

[4] A. H. Cherin, Bell Labs., private communication.

[5] L. B. Jeunhomme, Single-Mode Fiber Optics. New York: Marcel Dekker, 1983.

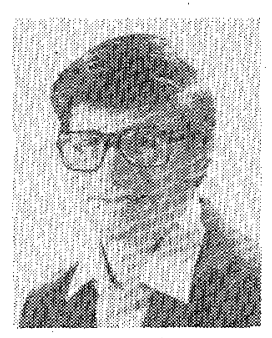

Douglas L. Franzen (S'64-M'66) was born in Minneapolis, MN, in 1942. He attended the University of Minnesota, Minneapolis, where he received the B.S., M.S.E.E., and Ph.D. degrees in electrical engineering in 1964, 1966, and 1970 , respectively, with partial support from an NSF grant.

From 1970 to 1972 he was an NRC/NBS postdoctoral Research Fellow in the Quantum Electronics Division at the National Bureau of Standards, Boulder, $\mathrm{CO}$, where he performed some of the early work on continuous laser sustained plasmas. Since then he has been with NBS where he has developed NBS standard instruments for high-power laser calorimetry and has shared in some original work on sputtered metal vapor ion lasers. His most recent interests include optical-fiber waveguide metrology and associated technologies. Currently he is a chairman of an EIA committee on the interlaboratory testing of optical-fiber measurement procedures, and an organizer of the biennial NBS Symposium on Optical Fiber Measurements.

Dr. Franzen is a member of the Optical Society of America. He was awarded the Dept. of Commerce bronze and silver medals for his effort in optical-fiber measurements. 